\section{Globalización y enfermedades infecciosas en las poblaciones indígenas de México}

\author{
Globalization and infectious diseases in Mexico's \\ indigenous population
}

\footnotetext{
${ }^{1}$ Centro Regional de Investigaciones Multidisciplinarias, Universidad Nacional Autónoma de México, Cuernavaca, México. 2 Centro de Investigación en Sistemas de Salud, Instituto Nacional de Salud Pública, Cuernavaca, México.

Correspondencia R. Castro

Centro Regional de Investigaciones Multidisciplinarias, Universidad Nacional Autónoma de México. Apartado Postal 4-106, C.P. 62431, Cuernavaca, Morelia, México. rcastro@servidor.unam.mx
}

\begin{abstract}
This paper discusses the health status of indigenous populations in Mexico. The first section characterizes the concept of globalization and its links to the population's health. Based on available statistical data, the second section documents the current indigenous populations' health status in the country. The article then argues that the presupposition of equity, crucial to globalization theory, does not apply to this case. Using the Mexican National Health Survey (2000), the third section further analyzes the health status of indigenous populations and identifies important inconsistencies in the data. The discussion section contends that these inconsistencies derive from the fact that such health surveys fail to contemplate the cultural specificities of indigenous peoples, thus leading to erroneous interpretations of the data. The article concludes that statistics on indigenous peoples' health must be interpreted with extreme caution and always with the support of social science theories and research methods.
\end{abstract}

Communicable Diseases; Indigenous Health; Equity
Roberto Castro 1

Joaquina Erviti 1

René Leyva 2

\section{Globalización}

El tema de la "globalización" es a la vez familiar y complejo. El uso frecuente de la expresión - en los medios de comunicación, en los discursos políticos, en el espacio académico, por mencionar sólo algunos - ha generado una suerte de certeza colectiva, por lo menos entre las capas medias y altas de la sociedad, respecto a la vigencia del fenómeno. El concepto alude simultáneamente a por lo menos tres dimensiones de la experiencia cotidiana: primero, la globalización "explica" la creciente sensación de que el mundo se ha reducido de tamaño, sensación que a su vez se deriva, entre otras cosas, del desarrollo de los medios de comunicación y en particular de Internet; segundo, el concepto también "explica" que muchas de nuestras acciones políticas, económicas, y de otros tipos, otrora locales, tengan ahora un carácter de mucho más amplio alcance, más allá de nuestras propias fronteras; y tercero, el concepto de "globalización" también hace referencia a una "tendencia mundial" - de corte fundamentalmente económico, pero también político, social, ambiental, y de salud - frente a la que aparentemente no hay nada que hacer, ante la que los individuos tienen escaso o nulo control, y a la que más vale sumarse. En este sentido, globalización se refiere "no a lo que nosotros queremos hacer, sino a lo que nos sucede a todos" (cursivas en el original) ${ }^{1}$ (p. 81). 
El tema de la globalización es objeto de un intenso debate internacional en el que se escuchan posiciones que destacan tanto los beneficios como las desventajas del fenómeno. La dificultad en seguir este debate estriba, entre otras cosas, en que hay por lo menos seis temas que habitualmente se asocian con la globalización, a saber: (a) la cuestión tecnológica, referida al desarrollo de los medios y sistemas de comunicación; (b) la cuestión económica, referida a la expansión global del capitalismo (aumento del volumen y frecuencia de los flujos de capital, bienes y divisas, privatizaciones, etc.); (c) la cuestión socio-cultural, referida al fortalecimiento de la sociedad civil, al flujo e intercambio de ideas, y a la occidentalización del conocimiento de los pueblos indígenas; (d) la cuestión demográfica, referida al incremento en los desplazamientos de personas por el mundo; (e) la cuestión política, referida a la creciente preocupación mundial por la democracia, los derechos humanos, y a la necesidad de impulsar un mayor número de acuerdos multilaterales; y (f) la cuestión medioambiental, referida a la creciente preocupación mundial por la degradación del medio ambiente, el cambio climático global, y el interés por preservar los bienes públicos globales 2 .

A los efectos de este artículo, suscribimos el concepto de globalización que hace referencia al creciente proceso de desregulación económica y financiera que está teniendo lugar en el mundo y cuya consecuencia inmediata es un creciente "flujo de información, bienes, capital y personas a través de las fronteras económicas y políticas" 3 (p. 22). El proceso de globalización de cada país va acompañado de un conjunto de reformas tales como privatizaciones, liberalización del comercio, desregulación financiera interna, desregulación financiera internacional, reforma en materia fiscal y de transferencias, y reforma del mercado laboral.

Walt 4 ha identificado diversas consecuencias en el ámbito de la salud que se derivan del proceso de globalización. Por ejemplo, se está dando un impulso a los mercados de salud (hospitales, seguros) con inversiones "seguras" y abandono de los sectores más pobres; la industria farmacéutica se está desarrollando con un nuevo impulso, con frecuencia bajo prioridades de producción según la demanda del mercado y no según necesidades reales; una nueva forma de relación entre los sectores público y privado en salud, donde la participación de este último se ha visto fortalecida por la mercantilización y/o privatización de bienes y servicios en salud, otrora reservados para el sector público; y finalmente un incremento en las enfermedades infecciosas particularmente entre los pobres, consecuencia del aumento de la desigualdad entre pobres y ricos 5 .

Este último aspecto, el de las enfermedades infecciosas, es el que nos interesa destacar en este trabajo, en relación con la población indígena de México. La globalización, como han sugerido diversos autores, implica mucho más que la mera apertura de los mercados y la desregulación financiera internacional. La globalización entraña una radicalización del capitalismo, con una consecuente exacerbación de sus efectos. Permite a las clases medias y altas incrementar tanto su nivel como su velocidad de consumo. Pero refuerza, por la propia lógica del mercado, la cultura de lo efímero (primacía de lo desechable y de lo reemplazable), de lo superfluo (primacía de productos innecesarios), y de lo precario (primacía de la inestabilidad laboral, cancelación del concepto tradicional de "carrera laboral", etc.) 6 .

La teoría de la globalización supone condiciones de inicio eficientes y equitativas entre los diversos países (mercados nacionales competitivos, baja concentración de la riqueza, acceso universal a los servicios de salud, etc.) con el fin de asegurar una distribución relativamente equitativa de sus beneficios. Los resultados a que ha dado lugar la globalización en los diversos países están claramente asociados a las condiciones económicas, sociales y ambientales pre-existentes, así como al tipo de políticas (de salud, educación y bienestar) que se han adoptado para fortalecer las capacidades ciudadanas 7,8. Existen estudios que muestran que, en términos macroeconómicos al menos, la globalización ha sido benéfica para algunos países, particularmente del sudeste asiático, que pudieron impulsar su participación en el comercio mundial replicando en buena medida el patrón de desarrollo que siguieron las economías más ricas del mundo 9, a través de medidas políticas y económicas que hoy prohíbe la Organización Mundial del Comercio.

Por ello, la experiencia de la globalización económica ha resultado enteramente diferente para otros países donde existen condiciones de menor desigualdad social. Las desventajas, como cabe esperar, se han concentrado sobretodo en los grupos más desfavorecidos, particularmente las poblaciones indígenas, que han vivido en buena medida excluidas de los modelos de desarrollo económico y social impulsados durante las últimas décadas.

En lo que sigue caracterizaremos brevemente las condiciones sociales en las que la globalización encuentra a las poblaciones indígenas de México; después discutiremos la situación de salud de los indígenas de este país, particularmente en relación a las enfermedades infecciosas, y lo haremos en comparación con la población no 
indígena. Ello nos permitirá, en primer lugar, argumentar que existen datos concretos que documentan el rezago en el que la globalización encuentra a los indígenas de este país, incumpliéndose, por lo tanto, el presupuesto fundamental de la teoría de los beneficios de la globalización; pero en segundo lugar, argumentaremos que la calidad misma de los datos sobre salud de los indígenas estará en entredicho siempre que no se atienda a otras consideraciones, de carácter sociocultural, que son indispensables en el estudio de la salud de estos pueblos; y que, consecuentemente, la capacidad de analizar y de intervenir en los procesos de salud de los pueblos indígenas en el marco de la globalización requiere de un sistemático proceso de evaluación de la información y de la generación de nuevos indicadores con sensibilidad cultural que expresen más fidedignamente la realidad.

\section{Indígenas en México: condiciones de vida y salud según los datos disponibles}

La marginalidad de los indígenas en este país se expresa incluso en la dificultad metodológica que presentan los censos y las encuestas nacionales en lo que se refiere a la diferenciación de la población indígena de la no-indígena (el problema de la calidad de los datos para estudiar a las poblaciones indígenas ha sido reportado en diversos ámbitos 10). Lo cual a su vez repercute en la estimación que se puede hacer sobre el tamaño real de la población indígena. Existe en la actualidad un debate en México acerca de los criterios que deben considerarse para definir esta población. Las posibilidades van desde considerar únicamente a la población que habla alguna lengua indígena (6,3 millones, equivalentes al 6,5\% del total de los mexicanos), hasta considerar como indígenas a estos más todos los habitantes de los hogares donde vive al menos una persona hablante de lengua indígena, y "corrigiendo" por diversas variables. En este último caso el total oscila entre los 12 y 13 millones de mexicanos (entre $12 \%$ y $13 \%$ de la población total) 11,12,13. La Encuesta Nacional de Salud 2000 14, realizada en el año 2000, identificó una proporción de $8 \%$ de población indígena a partir de la pregunta de si la persona entrevistada habla alguna lengua indígena, dato que coincide con el reportado en el último Censo de Población 15. Por lo tanto, en lugar de hablar de población indígena, hablaremos de población hablante de alguna lengua indígena.

La condición de pobreza de la población hablante de alguna lengua indígena en este país es bien conocida. El 88\% de los 803 municipios in- dígenas (municipios con $70 \%$ o más de población hablante de alguna lengua indígena) del país se encuentran en condiciones de alta y muy alta marginación. Casi un tercio (32\%) de la población hablante de alguna lengua indígena de este país se concentra en los estados de Chiapas, Oaxaca y Guerrero, estados en los que a su vez sólo vive alrededor del $11 \%$ de la población total del país 16 . Menos del 5\% de las localidades indígenas de México cuentan con los servicios urbanos básicos 17. En 1995, alrededor del 86\% de las viviendas mexicanas disponían de agua; entre los hablantes de alguna lengua indígena, este porcentaje no llegaba a $63 \%$; de igual forma, en ese mismo año el 93\% de las viviendas del país contaba con energía eléctrica, y 75\% disponía de drenaje; entre los hablantes de alguna lengua indígena estos porcentajes eran de sólo $75 \%$ y $34 \%$ respectivamente. Por otra parte, mientras que en el país sólo el $22 \%$ de la fuerza laboral se dedica a la agricultura, entre las comunidades indígenas este porcentaje asciende al $70 \% 18$.

Los estados de Oaxaca y Chiapas, localizados en el sureste del país, concentran al $25 \%$ de la población hablante de alguna lengua indígena de este país, y presentan un alto grado de marginación. En contraste, el estado de Nuevo León, localizado en la zona fronteriza con los Estados Unidos, no reúne ni el 1\% de la población hablante de alguna lengua indígena del país, y presenta uno de los niveles más avanzados de desarrollo económico y social 19. No sorprende, por ello, que mientras en Oaxaca y Chiapas sólo el 13\% de la población hablante de alguna lengua indígena tiene más de seis años de escolaridad, en Nuevo León este porcentaje llega al 66\% (entre población no-hablante de alguna lengua indígena). Mientras que en Oaxaca y Chiapas el $44 \%$ de la población hablante de alguna lengua indígena mayor de 25 años tiene más tres hijos, en Nuevo León este porcentaje sólo llega al 27\% 16.

En términos de salud, cabe destacar que en 1998 la esperanza de vida al nacer de la población nacional era de 74 años, mientras que entre la población hablante de alguna lengua indígena era de sólo 69 años. De igual forma, los datos disponibles indican que la mortalidad infantil en la población hablante de alguna lengua indígena es $58 \%$ superior a la media nacional 20 . Otros datos que ilustran el rezago de la población hablante de alguna lengua indígena son aún más dramáticos: en el estado de Guerrero, la tasa de mortalidad infantil es de 52 por 1.000, mientras que en Nuevo León la tasa es de 14 por 1.000. Si contrastamos los municipios más diferenciados tenemos que mientras que en San Nicolás de los Garza (Nuevo León) la tasa de mortalidad infantil es de 9 por 1.000, en San Martín Huamelulpan (Oaxaca) la 
cifra llega a 103 21. Por otra parte, el número de muertes maternas entre mujeres hablantes de alguna lengua indígena es casi del triple, en comparación con las mujeres no-hablantes de alguna lengua indígena 17.

Como señala con toda claridad el Programa Nacional para el Desarrollo de los Pueblos Indígenas 2001-2005: "tres de las principales causas de muerte entre la población indígena son las infecciones intestinales, la gripe y la neumonía, enfermedades cuyos condicionantes básicos son el saneamiento, la calidad de la vivienda, y los condicionantes microambientales de éstas..." 17 (p. 43). La Tabla 1 permite comparar las tasas de mortalidad estandarizadas por edad de las enfermedades infecciosas intestinales, las infecciones respiratorias agudas y la tuberculosis pulmonar entre los estados de Chiapas y Oaxaca (cuya población hablante de alguna lengua indígena constituye el 38\% del total de la población de esos estados), y Nuevo León (cuya población no-hablante de alguna lengua indígena constituye el 99,2\% de la población de esa entidad). Se aprecia ahí que existe un marcado contraste entre la mortalidad por estas causas en Chiapas y Oaxaca, por un lado, y Nuevo León por otro. En todos los casos, Chiapas representa el estado con la más alta tasa de mortalidad (excepto en el caso de las IRAs entre hombres, donde ocupa el segundo lugar entre 32). Le sigue Oaxaca que tiende a ocupar el segundo lugar, salvo en el caso de las IRAs entre hombres (octavo lugar) y la tuberculosis pulmonar entre mujeres (22). El estado de Nuevo León, en contraste, presenta consistentemente tasas comparativamente bajas de mortalidad por estas causas.

En síntesis, la información disponible muestra con contundencia lo que bien sabemos: que la condición social y de salud de los pueblos ha- blantes de alguna lengua indígena se caracteriza por un enorme rezago y por la presencia de enfermedades evitables. Pero la "información disponible” también puede ser problemática, y sobre sus implicaciones queremos reflexionar ahora.

\section{Las estadísticas desconcertantes}

La Tabla 2 presenta una comparación entre la población hablante de alguna lengua indígena y la no-hablante de alguna lengua indígena de México, en función de ciertos indicadores básicos de salud recogidos por la Encuesta Nacional de Salud 2000 14. Se aprecia ahí que, según los datos de esta fuente, una mayor proporción de personas $(13,6 \%)$ entre la población no-hablante de alguna lengua indígena de México reportó haber padecido alguna enfermedad en las dos semanas previas a la encuesta, en comparación con la proporción de personas hablante de alguna lengua indígena $(9,57 \%)$ que reportó lo mismo $(\mathrm{p}=0,00)$. En ambos grupos de población, la proporción de personas que habiendo enfermado en las últimas dos semanas utilizó algún servicio médico moderno fue semejante (58\%). Entre aquellos que reportaron haber sufrido alguna enfermedad en esas dos semanas, la proporción de infecciones en comparación con padecimientos crónicos y de otro tipo, fue también significativamente mayor $(p=0,00)$ entre la población no-hablante de alguna lengua indígena (53\%), en comparación con la población hablante de alguna lengua indígena (43\%). Estos datos reflejan un sub-registro, debido a que la pregunta sobre si habla o no alguna lengua indígena no se aplicó a menores de 5 años. Cualquier tabulación que controle por condición indígena, por lo tanto, necesariamente excluye a ese grupo de población. Tratándose

Tabla 1

Tasa de mortalidad (estandarizada por edad) por tres tipos de enfermedades transmisibles en Chiapas, Oaxaca,

Nuevo León, México, y a nivel nacional por sexo, 2001.

\begin{tabular}{|c|c|c|c|c|c|c|}
\hline \multirow[t]{2}{*}{ Entidad Federativa } & \multicolumn{2}{|c|}{$\begin{array}{l}\text { Enfermedades infecciosas } \\
\text { intestinales }\end{array}$} & \multicolumn{2}{|c|}{$\begin{array}{c}\text { Infecciones respiratorias } \\
\text { agudas }\end{array}$} & \multicolumn{2}{|c|}{ Tuberculosis pulmonar } \\
\hline & Mujeres & Hombres & Mujeres & Hombres & Mujeres & Hombres \\
\hline Chiapas & $18,8(1)$ * & $18,9(1)$ & $25,1(1)$ & $29,9(2)$ & $10,1(1)$ & $14,8(1)$ \\
\hline Oaxaca & $13,1(2)$ & $13,9(2)$ & $21,4(2)$ & $22,9(8)$ & $1,8(22)$ & $10,3(2)$ \\
\hline Nuevo León & $2,7(26)$ & $2,5(28)$ & $10,5(26)$ & $16,5(17)$ & $2,8(12)$ & $6,1(18)$ \\
\hline Nacional & 5,5 & 5,8 & 13,4 & 19,8 & 4,8 & 5,9 \\
\hline
\end{tabular}

* El número entre paréntesis indica el lugar que ocupa la entidad federativa en el conjunto de los 32 estados del país.

Fuente: Sistema Nacional de Salud 23 
Comparación entre la población hablante de lengua indígena y la no-hablante de lengua indígena de México en general, de Oaxaca y Chiapas, de Hidalgo y San Luis Potosí, y de Chiapas-Oaxaca y Nuevo León, a partir de los datos de la Encuesta Nacional de Salud 2000.

\begin{tabular}{|c|c|c|c|c|c|c|c|c|c|c|c|}
\hline & $\begin{array}{c}\text { A } \\
\text { Población } \\
\text { hablante } \\
\text { de lengua } \\
\text { indígena * } \\
\text { de México }\end{array}$ & $\begin{array}{c}\text { B } \\
\text { Población } \\
\text { no hablante } \\
\text { de lengua } \\
\text { indígena de } \\
\text { México }\end{array}$ & $\begin{array}{l}\text { A-B * } \\
(p)\end{array}$ & $\begin{array}{c}\text { D } \\
\text { Población } \\
\text { hablante } \\
\text { de lengua } \\
\text { indígena de } \\
\text { Chiapas y } \\
\text { Oaxaca }\end{array}$ & $\begin{array}{c}\text { E } \\
\text { Población } \\
\text { no-hablante } \\
\text { de lengua } \\
\text { indígena de } \\
\text { Chiapas y } \\
\text { Oaxaca }\end{array}$ & $\begin{array}{c}\text { D-E ** } \\
(p)\end{array}$ & $\begin{array}{c}\text { G } \\
\text { Población } \\
\text { hablante } \\
\text { de lengua } \\
\text { indígena } \\
\text { de Hidalgo } \\
\text { y San Luis } \\
\text { Potosí }\end{array}$ & $\begin{array}{c}\text { H } \\
\text { Población } \\
\text { no-hablante } \\
\text { de lengua } \\
\text { indígena } \\
\text { de Hidalgo } \\
\text { y San Luis } \\
\text { Potosí }\end{array}$ & $\begin{array}{c}\text { G-H *** } \\
(p)\end{array}$ & $\begin{array}{c}\text { J } \\
\text { Población } \\
\text { no-hablante } \\
\text { de lengua } \\
\text { indígena } \\
\text { de Nuevo } \\
\text { León }\end{array}$ & $\begin{array}{l}\text { D-J \# } \\
(p)\end{array}$ \\
\hline $\begin{array}{l}\text { Población } \\
\text { que declaró } \\
\text { haber pade- } \\
\text { cido de alguna } \\
\text { enfermedad } \\
\text { en las últimas } \\
\text { dos semanas }\end{array}$ & $\begin{array}{c}9,57 \\
(1.277 / \\
12.060)\end{array}$ & $\begin{array}{c}13,6 \\
(20.920 / \\
132.943)\end{array}$ & $(0,00)$ & $\begin{array}{c}8,71 \\
(379 / \\
3.973)\end{array}$ & $\begin{array}{c}11,24 \\
(690 / \\
5.448)\end{array}$ & $\begin{array}{c}17,8493 \\
(0,00)\end{array}$ & $\begin{array}{c}9,29 \\
(170 / \\
1.659)\end{array}$ & $\begin{array}{c}12,82 \\
(1.282 / \\
7.840)\end{array}$ & $\begin{array}{c}30 \\
(0,00)\end{array}$ & $\begin{array}{c}12,78 \\
(670 / \\
4.572)\end{array}$ & $\begin{array}{c}40,5 \\
(0,00)\end{array}$ \\
\hline $\begin{array}{l}\text { Proporción } \\
\text { de infecciones } \\
\text { (vs. otros pa- } \\
\text { decimientos) }\end{array}$ & $\begin{array}{l}43,3 \\
(552 / \\
722)\end{array}$ & $\begin{array}{c}52,7 \\
(10.991 / \\
9.863)\end{array}$ & $\begin{array}{c}42,3 \\
(0,00)\end{array}$ & $\begin{array}{c}51,72 \\
(195 / 182)\end{array}$ & $\begin{array}{c}47, .82 \\
(329 / 359)\end{array}$ & $\begin{array}{c}1,4854 \\
(0,22)\end{array}$ & $\begin{array}{c}34,12 \\
(58 / 112)\end{array}$ & $\begin{array}{c}53,24 \\
(681 / 598)\end{array}$ & $\begin{array}{l}21,97 \\
(0,00)\end{array}$ & $\begin{array}{c}40,06 \\
(268 / 401)\end{array}$ & $\begin{array}{c}13,3 \\
(0,00)\end{array}$ \\
\hline $\begin{array}{l}\text { Población } \\
\text { que percibe } \\
\text { su estado de } \\
\text { salud como } \\
\text { "bueno" o } \\
\text { "muy bueno" }\end{array}$ & $\begin{array}{c}56,8 \\
(7.573 / \\
5.772)\end{array}$ & $\begin{array}{c}40,9 \\
(90.708 / \\
62.854)\end{array}$ & $\begin{array}{c}27,3 \\
(0,00)\end{array}$ & $\begin{array}{c}60,9 \\
(2.648 / \\
1.699)\end{array}$ & $\begin{array}{c}53,77 \\
(3.284 / \\
2.824)\end{array}$ & $\begin{array}{l}52,89 \\
(0,00)\end{array}$ & $\begin{array}{c}55,5 \\
(1.019 / \\
816)\end{array}$ & $\begin{array}{c}56,43 \\
(5.140 / \\
3.968)\end{array}$ & $\begin{array}{c}0,51 \\
(0,48)\end{array}$ & $\begin{array}{c}71,5 \\
(3.743 / \\
1.492)\end{array}$ & $\begin{array}{l}119,8 \\
(0,00)\end{array}$ \\
\hline $\begin{array}{l}\text { Proporción } \\
\text { de derecho- } \\
\text { habientes de } \\
\text { seguridad } \\
\text { social (vs. } \\
\text { usuarios de } \\
\text { servicios pri- } \\
\text { vados u otros } \\
\text { y población } \\
\text { no cubierta) }\end{array}$ & $\begin{array}{c}16,7 \\
(2.225 / \\
11.095)\end{array}$ & $\begin{array}{c}43,9 \\
(65.945 / \\
8.951)\end{array}$ & $\begin{array}{c}3,47 \\
(0,00)\end{array}$ & $\begin{array}{c}5,26 \\
(229 / \\
4.123)\end{array}$ & $\begin{array}{c}26,07 \\
(16.03 / \\
4.545)\end{array}$ & $\begin{array}{c}766,26 \\
(0,00)\end{array}$ & $\begin{array}{c}10,19 \\
(186 / 1.640)\end{array}$ & $\begin{array}{c}33,13 \\
(3.015 / \\
6.086)\end{array}$ & $\begin{array}{c}386,47 \\
(0,00)\end{array}$ & $\begin{array}{c}67,48 \\
(3.536 / \\
1.604)\end{array}$ & $\begin{array}{r}3974,7 \\
(0,00)\end{array}$ \\
\hline $\begin{array}{l}\text { Población } \\
\text { que reportó } \\
\text { algún pade- } \\
\text { cimiento en } \\
\text { las últimas } \\
\text { dos semanas } \\
\text { y que utilizó } \\
\text { servicios de } \\
\text { salud (vs. } \\
\text { autoatención } \\
\text { u otros servi- } \\
\text { cios) }\end{array}$ & $\begin{array}{c}58,4 \\
(744 / 530)\end{array}$ & $\begin{array}{c}57,7 \\
(12.012 / \\
8.801)\end{array}$ & $\begin{array}{c}0,23 \\
(-0,63)\end{array}$ & $\begin{array}{c}49,47 \\
(187 / 191)\end{array}$ & $\begin{array}{c}51,09 \\
(350 / 335)\end{array}$ & $\begin{array}{c}0,26 \\
(0,61)\end{array}$ & $\begin{array}{c}58,58 \\
(99 / 70)\end{array}$ & $\begin{array}{c}58,8 \\
(745 / 522)\end{array}$ & $\begin{array}{l}0,003 \\
(0,96)\end{array}$ & $\begin{array}{c}63,92 \\
(427 / 241)\end{array}$ & $\begin{array}{c}20,8 \\
(0,00)\end{array}$ \\
\hline
\end{tabular}


de enfermedades infecciosas, dicha exclusión resulta de suma trascendencia, pues es bien sabido que la mayor parte de las infecciones se presentan en menores de cinco años de edad. En el año 2000 , el $73 \%$ de los casos nuevos atendidos en consulta externa de la población menor de un año residente en municipios con predominio de habla indígena fue debido a enfermedades infecciosas respiratorias. Dicha proporción fue de $64 \%$ para población de entre 1 a 4 años en los mismos municipios, y de $58 \%$ para la población de todos los grupos de edad también en los mismos municipios 22. Congruente con este dato, un mayor porcentaje (57\%) de integrantes de la población hablante de alguna lengua indígena reportó percibir su estado de salud como "bueno" o "muy bueno" en comparación con los no-hablantes de alguna lengua indígena del país $(41 \%)(\mathrm{p}=0,00)$. Por tanto, los datos parecen contradecir lo que de otra manera sabemos: que la situación de los hablantes de alguna lengua indígena de este país es, en general, peor que la de la población no-hablante de alguna lengua indígena. Más aún cuando, de acuerdo a la misma Tabla 2, la proporción de población hablante de alguna lengua indígena cubierta por la seguridad social es de apenas cerca del $17 \%$, mientras que a nivel nacional esta proporción es de casi $44 \%(\mathrm{p}=0,00)$. ¿A qué podemos atribuir la disparidad encontrada en los datos de la Encuesta Nacional de Salud 2000 14, según la cual la morbilidad entre los hablantes de alguna lengua indígena de México en general es menor que la de la población no-hablante de alguna lengua indígena del país?

Una estrategia en busca de respuesta a esta pregunta es el examen de los datos en submuestras, que permitan replicar el análisis anterior pero a partir de los datos de poblaciones más específicas. La Tabla 2 permite comparar a las poblaciones hablantes de alguna lengua indígena y no-hablantes de alguna lengua indígena, tanto de los estados de Chiapas y Oaxaca, como de los estados de Hidalgo y San Luis Potosí; así como a dos poblaciones que se ubican en extremos opuestos en términos de indicadores de desarrollo: la población hablante de alguna lengua indígena de los estados de Oaxaca y Chiapas, y la población no-hablante de alguna lengua indígena del estado de Nuevo León. Como argumentaremos en seguida, sugerimos que estos datos - los que comparan dos extremos de nuestra realidad - reflejan mejor la condición de los hablantes de alguna lengua indígena que los datos agregados de la encuesta nacional en su conjunto.

El primer dato que llama la atención es la relativa semejanza que existe en los porcentajes de población hablante de alguna lengua indígena y no-hablante de alguna lengua indígena, a lo lar- go de la tabla, que declararon haber padecido alguna enfermedad en las últimas dos semanas: alrededor de $9 \%$ en el primer caso, y alrededor de $12 \%$ en el segundo caso (siempre con $\mathrm{p}=0,00$ ), dato que parece confirmar que, según la Encuesta Nacional de Salud 2000, la población hablante de alguna lengua indígena de este país reporta menores índices de morbilidad que la población no-hablante de alguna lengua indígena.

Un dato particularmente importante para esclarecer esta cuestión se refiere a la proporción de derechohabientes de la seguridad social existente en ambos tipos de población (hablante de alguna lengua indígena y no-hablante de alguna lengua indígena): la proporción de población nohablante de alguna lengua indígena asegurada es 2,6 veces mayor que la población hablante de alguna lengua indígena a nivel nacional; pero dicha razón crece a 3,25 veces en los estados de Hidalgo y San Luis Potosí, y a 4,9 en los estados de Oaxaca y Chiapas. La razón se incrementa hasta 12,83 ahí donde la comparación se efectúa entre dos tipos de población ubicados en extremos opuestos: la población hablante de alguna lengua indígena de Chiapas y Oaxaca, y la población no-hablante de alguna lengua indígena de Nuevo León (sólo poco más del 5\% de la población hablante de alguna lengua indígena en Chiapas y Oaxaca, dato que contrasta severamente con el $67 \%$ de la población de Nuevo León). Estas proporciones son sólo la expresión radicalizada de la misma tendencia que ya habíamos apreciado en relación a la muestra nacional.

Un dato adicional se refiere a la proporción de población que efectivamente utilizó algún servicio de salud una vez que se enfermó (en las últimas dos semanas). Tanto a nivel nacional, como al interior de los estados de Chiapas/Oaxaca e Hidalgo/San Luis Potosí, el porcentaje de utilización de servicios entre hablantes de alguna lengua indígena y no-hablantes de alguna lengua indígena en cada uno de estos escenarios fue prácticamente idéntico. Sólo al comparar a poblaciones extremas aparece una diferencia importante: la utilización de servicios fue mucho más alta en Nuevo León (casi 64\%), en comparación con la población hablante de alguna lengua indígena de Chiapas y Oaxaca (sólo 50\%; $\mathrm{p}=0,000$ )

La utilización de servicios, naturalmente, está determinada en primer lugar por la disponibilidad de los mismos. De acuerdo al Programa Nacional de Salud 2001-2006, en las zonas indígenas de Oaxaca el número de médicos por 1.000 habitantes hablante de alguna lengua indígena es de 0,13 , frente a 0,94 en el estado en su conjunto, y 1,38 a nivel nacional; en Chiapas el número de médicos por 1.000 habitantes es de 1,05 a nivel 
estatal, y en Nuevo León es de 1,54 20,23. Lo mismo ocurre con las camas censables: en las zonas indígenas de Oaxaca la cifra es de 0,07 , a nivel estatal es de 4,91, mientras que a nivel nacional es de 7,91 por 10.000 habitantes; en Chiapas la cifra estatal es de 4,67, mientras que en Nuevo León es de 9,56 20,23.

\section{Discusión}

Como ha sido documentado en la literatura sobre medicalización 24,25,26 la presencia de servicios de salud contribuye decisivamente a la propagación de un modo específico de entender la salud y la enfermedad. La expansión de la medicina moderna no sólo supone la presencia de servicios de salud en zonas cada vez más amplias, sino que incluye también la socialización de conceptos y categorías de conocimiento, de códigos específicos, que permiten identificar y nombrar a la enfermedad. La hipótesis que proponemos es que la medicalización occidental le da sentido a los de otra manera extraños datos de la Encuesta Nacional de Salud 2000 14: los hablantes de alguna lengua indígena reportan haberse enfermado menos en las dos semanas previas a la encuesta que los habitantes no-hablantes de alguna lengua indígena en todo el país, porque las categorías y conceptos usados en dicha encuesta les son culturalmente ajenos; y por la misma razón - porque la distancia de la medicina moderna con respecto a los pueblos indios se manifiesta también en la escasa presencia de servicios adecuados de salud en sus comunidades - los hablantes de alguna lengua indígena de Chiapas y Oaxaca reportan haber utilizado menos los servicios de salud en comparación con los habitantes no-hablantes de alguna lengua indígena de Nuevo León. El efecto de contraste entre extremos se pierde al utilizar la muestra nacional en su conjunto, o submuestras relativamente homogeneizadas (como las de Chiapas/Oaxaca e Hidalgo/San Luis Potosí). De ahí que en estos otros escenarios la utilización aparece como equivalente entre indígenas y no indígenas.

En este marco, entonces, es posible explicar el comportamiento de la variable "percepción del propio estado de salud como bueno o muy bueno" en los diferentes escenarios. Obsérvese que tanto a nivel nacional, como en el interior de los estados de Chiapas y Oaxaca, es mayor la proporción de hablantes de alguna lengua indígena que perciben su estado de salud positivamente, en comparación con los no-hablantes de alguna lengua indígena. Pero mientras que en el primer caso la razón entre ambas proporciones es de 1,39 a favor de los hablantes de alguna lengua indígena, en el segundo dicha razón se reduce a 1,13. En el tercer caso (Hidalgo/San Luis Potosí), en cambio, la diferencia entre ambas poblaciones desaparece del todo. Y en el cuarto, ahí donde comparamos a dos poblaciones extremas, la tendencia se ha revertido: el porcentaje de población no-hablante de alguna lengua indígena de Nuevo León que percibe su salud positivamente es 1,17 veces mayor que el porcentaje de la población hablante de alguna lengua indígena de Chiapas y Oaxaca.

Proponemos que así se explica que a la pregunta de cómo perciben su propia salud, la proporción de individuos que la consideran "buena" o "muy buena" sea menor entre los indígenas de Chiapas y Oaxaca (61\%) que entre los habitantes de Nuevo León (a pesar de haber reportado una menor morbilidad en las dos semanas previas) (casi $72 \%$; $p=0,000$ ). Pues una cosa es disponer en el propio acervo de cognoscitivo de las categorías de salud/enfermedad occidentales para poder dar cuenta de ellas en una estrategia de investigación - la encuesta - también occidental; y otra muy diferente es saber que se vive en desventaja, que se tiene una mala salud, independientemente de las categorías culturalmente específicas que se usen para nombrar esta experiencia.

Por otra parte, desde la antropología y otras disciplinas afines, se ha documentado que la condición de pobreza y marginación - como la que caracteriza a los indígenas de este país - impone umbrales de tolerancia frente al dolor y la enfermedad que son superiores a los de las capas medias urbanas 27 . Se trata, en buena medida, de una tolerancia impuesta por las propias condiciones de restricción y de dificultad de acceso a los servicios de salud.

Una fuente adicional de información de que disponemos, que muestra que las estrategias occidentalizadas de investigación en salud no siempre funcionan en contextos indígenas, se refiere a la reciente evaluación que se hizo en México sobre la calidad de los servicios de salud en el país. Utilizando indicadores de calidad fundados en la perspectiva de la medicina moderna (oportunidad de la atención, accesibilidad de los servicios, calidad del trato humano recibido, grado de satisfacción con la atención recibida, etc.), un estudio realizado por la Secretaría de Salud mostró que los niveles de calidad de los servicios de salud en zonas indígenas eran por lo menos equivalentes, o más altos que los de otras zonas del país 20. Los datos, por supuesto, sólo mostraban lo obvio: que los instrumentos de recolección de la información no servían. En otro trabajo 28 hemos argumentado que una de las razones de la inoperancia de las estrategias occidentales de in- 
vestigación en comunidades indígenas pasa por la discordancia de orientaciones cognoscitivas: la encuesta es un instrumento individualizado, que se contrapone a la práctica comunitaria de discusión típica de las comunidades indígenas. Lo que estamos proponiendo aquí es que las encuestas nacionales de salud, tal como han sido hechas hasta ahora, son sólo un instrumento muy limitado para conocer las condiciones de salud de las poblaciones indígenas. En la medida en que las encuestas de salud aplicadas en comunidades indígenas no se formulen tomando como referencia la manera en que dichas comunidades comprenden y elaboran sus procesos de salud-enfermedad, estaremos limitando nuestra capacidad real de medir tales procesos en escenarios de ese tipo.

La falta de atención a estas cuestiones, y la suposición de que los datos proporcionados por una encuesta son válidos per se, ha llevado a algunos autores a realizar análisis con base en la Encuesta Nacional de Salud 200014 realmente sorprendentes. Un estudio reciente del Banco Mundial 29, por ejemplo, pone en duda que existan barreras culturales entre la medicina moderna y las poblaciones indígenas de este país. Utilizando únicamente métodos estadísticos, los autores encuentran que vivir en ciudades representa una desventaja en comparación con vivir en comunidades indígenas, en lo que se refiere a la utilización de servicios preventivos de salud modernos - contrariamente a lo encontrado en otras investigaciones sobre la situación de los indígenas en zonas urbanas ${ }^{30}$; pues dicha utilización parece ser significativamente más alta en las comunidades que en las ciudades. Los autores incluso aventuran, como una explicación de este inesperado hallazgo, el probable éxito de programas oficiales de atención a los marginados, como los llamados Instituto Mexicano del Seguro Social (IMSS-Solidaridad), Programa de Educación Salud Y Alimentación (PROGRESA) y otros. Los autores afirman categóricamente: "cualesquiera que sean las razones de los anteriores hallazgos, la implicación es que sea cual fuere la desventaja cultural que pudo haber estado asociada con el hecho de ser rural e indigena, [dicha desventaja] ha sido largamente superada al menos en lo que se refiere a la atención preventiva moderna" 29 (p. 10).

El principal error de este tipo de análisis radica en su decisión de ignorar el conocimiento que sobre esta materia se ha acumulado dentro de las ciencias sociales, y en confiar ciegamente en el procesamiento estadístico de los datos. Si esa fuera nuestra postura, estaríamos obligados aquí a argumentar que, con base en la Encuesta Nacional de Salud 2000 14, podemos presumir que la salud de los indígenas de México presenta mejores condiciones que la de los habitantes no indígenas. No solamente no afirmamos tal cosa, sino que además, con base en dicho acervo de conocimientos, podemos formular hipótesis sobre la naturaleza de algunos de los datos de la Encuesta Nacional de Salud 2000, y sobre su correcta interpretación.

\section{Conclusiones}

El proceso de globalización no beneficia de manera homogénea a todos los países ni a todos los sectores de la sociedad. En el ámbito de la salud, y en particular con relación a los grupos más desfavorecidos como los indígenas, la globalización no es ajena a la permanencia y, en algunos casos, al resurgimiento de las enfermedades infecciosas. Ello es debido no sólo a factores ya bien identificados en la literatura, como el aumento del flujo en el comercio internacional, así como el aumento del movimiento de personas a través de las fronteras, fenómenos que crean las condiciones necesarias para la propagación de enfermedades infecciosas 2 . La permanencia y el resurgimiento de las enfermedades infecciosas, particularmente en poblaciones indígenas como las de México, también es resultado de la poca presencia política y del poco "peso epidemiológico" de dichos padecimientos. La carga de la deuda externa ha obligado a una permanente reducción del gasto social, el de salud incluido. Los limitados presupuestos, y la presencia de otros padecimientos también importantes, pero que además se acompañan de un creciente activismo político por parte de grupos sociales específicos, colocan el problema de las enfermedades infecciosas entre poblaciones indígenas en un lugar relativamente secundario dentro de las prioridades de política pública.

Esta "relativa invisibilidad" de la problemática de salud de los indígenas se asocia, además, con la falta de instrumentos de medición con sensibilidad cultural que se basen en las formas específicas en que las poblaciones indígenas dan cuenta de sus procesos de salud-enfermedad y que permitan medir, con mayor precisión, la prevalencia y los determinantes de sus padecimientos. Al respecto cabe señalar que desde 1996 se acordó en México que toda unidad de salud de primer nivel identificaría a los indígenas que asistan a recibir consulta. Esta medida tan elemental y relativamente sencilla de implementar, y que permitiría introducir la dimensión de $e t$ nicidad en las estadísticas de salud de este país, no ha sido aún puesta en práctica. Por ello, las estimaciones sobre la salud de la población indí- 
gena deben seguir haciéndose - como lo hemos mostrado aquí - a partir de estimaciones indirectas e inexactas.

Pero no basta con afinar técnicamente los instrumentos de recolección de la información. Hemos argumentado que una de las limitantes de la Encuesta Nacional de Salud 2000 - de su metodología, de su instrumentación - es que presupone una comprensión universal de los conceptos y de los padecimientos que investiga. Lo que hace falta, entonces, es nutrir este tipo de estrategias de investigación con el conocimiento acumulado en las ciencias sociales a lo largo de varias décadas. Es significativo lo que sabemos desde la sociología y la antropología sobre el horizonte cultural de muchas de las comunidades indígenas de nuestros países. Mucho de ese conocimiento (por ejemplo, sobre el significado de conceptos como "bienestar", "salud" y "enfermedad"; sobre la alienante experiencia de muchos indígenas al ser atendidos en una clínica de salud, etc.) podría potenciar de manera significativa nuestra capacidad de monitorear la evolución de los padecimientos infecciosos en esas poblaciones.

En la misma dirección, hay que señalar que en el marco de la globalización las políticas sociales se asientan en la individualización de riesgos y de alternativas: el caso del seguro popular en

\section{Resumen}

Se presenta un análisis sobre la salud de los indígenas de México. La primera parte analiza el concepto de globalización y busca identificar sus principales vínculos con la salud de las poblaciones. La segunda muestra, con base en datos estadísticos, el grado de rezago en que se encuentran las poblaciones indígenas y el incumplimiento del presupuesto de equidad, central en la teoría de los beneficios de la globalización. La tercera parte presenta un análisis de la salud de los indígenas en México basado en los datos de la Encuesta Nacional de Salud 2000 y muestra la existencia de importantes inconsistencias de esta información. En la sección de discusión se propone que tales inconsistencias derivan del hecho de que las mediciones estadísticas no contemplan las particularidades culturales de los pueblos indígenas, lo que lleva a interpretaciones erróneas. Se concluye que las estadísticas de salud de los indígenas deben ser valoradas con precaución y atendiendo a los desarrollos alcanzados en las ciencias sociales.

Enfermedades Transmisibles; Salud de la Población Indígena; Equidad
México es un ejemplo. La cuestión aquí es monitorear si junto a tales estrategias se están implementando otras de reducción de riesgos no individuales, sino de grupo; estrategias que apunten a mejorar las oportunidades y las condiciones de vida de los grupos indígenas.

En consecuencia, el problema de la permanencia de las enfermedades infecciosas en poblaciones indígenas se da en un marco caracterizado por: (a) una política globalizadora que presupone condiciones de equidad que no se cumplen para el caso de los indígenas; (b) una política pública que no necesariamente tiene a los indígenas como su prioridad fundamental, y que es influida por otros grupos sociales que, con más poder, han sido más exitosos en su activismo en torno a cuestiones de salud; y (c) un pobre monitoreo de sus condiciones de salud, resultado de estrategias de investigación pobres o inadecuadamente orientadas.

Se trata de tres condiciones - presupuestos de equidad no satisfechos, política pública que no prioriza a los más desfavorecidos, y prevalencia de diseños de investigación limitados - que, juntos, colocan el problema de los padecimientos infecciosos en poblaciones indígenas en un espacio muy definido: el espacio de la ética en salud.

\section{Colaboradores}

R. Castro planeó el estudio, elaboró la parte conceptual sobre globalización, y participó en la elaboración de la discusión y las conclusiones del artículo. J. Erviti elaboró el apartado sobre "Las Estadísticas Desconcertantes", participó en la elaboración de la discusión y las conclusiones del artículo, y sistematizó las referencias bibliográficas. R. Leyva generó y analizó la información sobre las condiciones de vida y salud de los indígenas en México, y participó en la elaboración de la discusión y las conclusiones del artículo. 


\section{Referencias}

1. Bauman Z. La globalización. consecuencias humanas. México DF: Fondo de Cultura Económica; 2003.

2. Maclean H, Labonte R, Glynn K, Sicchia SR. Globalization, gender and health: research to policy interface. Oklahoma City: Center for Research in Women's Health; 2003.

3. Daulaire N. Globalization and health. Development 1999; 42 (4 Suppl):22-4.

4. Walt, G. Globalization and health. http://www. phmovement.org/pubs/issuepapers/walt.html (accedido el 23/Mar/2006).

5. Programa de las Naciones Unidas para el Desarrollo. Informe sobre el desarrollo humano. New York: Programa de las Naciones Unidas para el Desarrollo; 1995.

6. Sennet R. La corrosión del carácter. Las consecuencias personales del trabajo en el nuevo capitalismo. Barcelona: Editorial Anagrama; 2000.

7. Cornia GA. Globalization and health: results and options. Bull World Health Organ 2001; 79 Suppl 9:834-41.

8. Programa de Naciones Unidas para el Desarrollo. Human development report 1999. New York: Programa de Naciones Unidas para el Desarrollo; 1999.

9. Rodriguez F, Rodrik D. Trade policy and economic growth: a skeptic's guide to the cross-national evidence. Boston: Harvard University Press; 2000.

10. McPherson KM, Harwood M, McNaughton HK. Ethnicity, equity, and quality: lessons from New Zealand. BMJ 2003; 327:443-4.

11. Corona R, Tuiran R. Tamaño de la población indígena mexicana. In: Consejo Nacional de Población, editores. La población de México en el nuevo siglo. México DF: Consejo Nacional de Población; 2001. p. 165-79.

12. Corona R. Indicadores censales a escala de hogares sobre población indígena. In: Taller Internacional Dinámica de la Población Indígena: Problemáticas Contemporáneas. México DF: Centro de Investigaciones y Estudios Superiores en Antropología Social; 2000.

13. Chávez A, Hernández H. La definición de la población indígena en México en el Censo de Población del año 2000. In: Encuentro sobre la Población en el Sureste de México. Tapachula: Sociedad Mexicana de Demografía; 2002.

14. Secretaría de Salud. Encuesta nacional de salud 2000. México DF: Secretaría de Salud/Instituto Nacional de Salud Pública; 2000.

15. Instituto Nacional de Estadística, Geografía e Informática. XII censo general de población y vivienda 2000. Aguascalientes: Instituto Nacional de Estadística, Geografía e Informática; 2001.

16. Carreto E, Embriz A, Fernández P, organizadores. Indicadores socioeconómicos de los pueblos indígenas de México, 2002. México DF: Instituto Nacional Indigenista/Programa de las Naciones Unidas para el Desarrollo en México/Consejo Nacional de Población; 2002.
17. Instituto Nacional Indigenista. Programa Nacional para el Desarrollo de los Pueblos Indígenas 20012006. México DF: Instituto Nacional Indigenista; 2001.

18. Instituto Nacional Indigenista/Programa de las Naciones Unidas para el Desarrollo/Instituto Nacional de Estadística, Geografía e Informática/Secretaría del Trabajo y Previsión Social/Secretaría de Desarrollo Social/Organización Internacional del Trabajo. Encuesta nacional de empleo en zonas indígenas 1997. México DF: Instituto Nacional de Estadística, Geografía e Informática; 1997.

19. Consejo Nacional de Población. Indicadores socioeconómicos e índices de marginación municipal 1990. México: Consejo Nacional de Población; 1993.

20. Secretaría de Salud. Programa nacional de salud 2001-2006. México DF: Secretaría de Salud; 2001.

21. Coordinación Nacional para los Pueblos Indígenas. Programa nacional para el desarrollo de los pueblos indígenas 2001-2006. México DF: Coordinación Nacional para los Pueblos Indígenas; 2001.

22. Investigación en Salud y Demografía. Diagnóstico sobre la salud de la población indígena, 2000. México DF: Investigación en Salud y Demografía; 2003.

23. Sistema Nacional de Salud. Recursos por entidad federativa. http://www.ssa.gob.mx/ (accedido el 01/Oct/2003).

24. Turner BS. Medical power and social knowledge. London: Sage Publications; 1987.

25. Conrad P, Schneider JW. Deviance and medicalization. From badness to sickness. Philadelphia: Temple University Press; 1992.

26. Lupton $\mathrm{D}$. The social construction of medicine and the body. In: Albrecht GL, Fitzpatrick R, Scrimshaw SC, editors. The handbook of social studies in health and medicine. Thousand Oaks: Sage Publications; 2000. p. 50-63.

27. Castro R. La vida en la adversidad. El significado de la salud y la reproducción en la pobreza. Cuernavaca: Centro Regional de Investigaciones Multidisciplinarias, Universidad Nacional Autónoma de México; 2000.

28. Castro R, Erviti J. Desarrollo de indicadores de calidad de la atención con sensibilidad intercultural. México DF: Secretaría de Salud; 2003. (Informe Técnico).

29. Paqueo VB, Gonzalez CY. Economic analysis of health care utilization and perceived illness: ethnicity and other factors. Policy Research Working Paper, 3125. http://econ.worldbank.org/files/ 29361_wps3125.pdf (accedido el 02/Abr/2006).

30. Vargas PN, Flores JI. Los indígenas en ciudades de México: el caso de los mazahuas, otomíes, triques, zapotecos y mayas. Papeles de Población 2002; 34:235-57

Recibido el 09/Nov/2005

Versión final presentada el 11/May/2006

Aprobado el 15/May/2006 